\title{
Consistency and Dynamics of the Pattern of Integration in ASEAN Stock Markets
}

\author{
David Kaluge, Suwandi* and Saqib Muneer \\ 'Brawijaya University, Indonesia; davidk@ub.ac.id, \\ ${ }^{2}$ Center of Postgraduate Studies, Cenderawasih University, Jayapura, Papua, Indonesia; \\ wandi1212@gmail.com, \\ ${ }^{3}$ Department of Business Administration, Government College University Faisalabad, Pakistan; \\ saqibmuneer85@gmail.com
}

\begin{abstract}
Objective: The pattern of integration is almost untouched topic in studies of capital market. This study aims at showing the pattern in ASEAN capital markets. Methodology: By using General Vector Auto Regression (GVAR), which includes many control variables of global capital markets, the study found that the integration in ASEAN markets is almost fully integrated. Except the relation between Philippines' capital Market and the Singapore's capital market, all other markets are fully integrated significantly. The integration exists at time zero (0), while at lag 1 and lag 2 periods it reduces sharply and become insignificant. Findings: These findings guide policy makers to pay more attention to any abnormal change in any ASEAN capital markets. A shock in any ASEAN market will easily and quickly affects all other markets in ASEAN region. Application: The care should be more on the markets that have both direction and single direction impact.
\end{abstract}

Keywords: ASEAN Capital Markets, Asymmetric Reaction, Abnormality Analysis, Bullish-Bearish Market, Contagion Effect, Leader Follower, Market Integration

\section{Introduction}

\subsection{Background}

The topic of capital market integration is common for discussion about capital market. Many studies have been done for this topic to identify how close the capital markets are in a specific region such as in Europe, South America or even in Asian region. All studies reached the same conclusion that the markets have been increasingly integrated. However, it is almost untouchable the topic of the characteristics of integration. Especially, hardly studies have been done to investigate the pattern of integration or the pattern of the relation among countries related in one integrated region. The identification of the pattern is considered significantly important because it could be asymmetrical effect among countries or even non-reciprocal. This study faced the challenge to identify the pattern which then is useful to make policy related to anticipate the external impact of other capital market in the region. This study took ASEAN region as the site for pattern analysis.

\subsection{Theoretical Discussion}

Capital market integration is basically a concept explaining that all capital markets are related each other. Due to the fact, if one market experiences a shock, it will easily transferred to other capital markets. This phenomenon is sometimes called as contagion effect. Contagion effect theories try to answer the question of whether the shock in a capital market will be transferred onto other capital markets.

The logic behind the contagion theories is simple. The effect from a market to others will happen if there are connections among them. The connection shows a special relation among them, and by some specific relation the effect will be transmitted. The connection will act as the channel of transmission. Many studies ${ }^{1-5}$ have discussed channels where contagion effect usually takes place. 
In 5 , 7 claims that by the change very rapidly in technology of information, Investors realize the fact that any change in any economy or any financial market will easily affect other capital markets and hence it will cause gain or loss related to their capital invested. This happens because all markets are linked technologically. Market integration could be explained by several theories. The first is business connection theory. In ${ }^{\beta, 9}$ states that in international trade, all business countries are connected by business relationship, export and import. The connection between exporting countries and importing ones implies that if an economic shock occurs in a country, his counterparts will also be affected. If industries in one country experience crisis, it will transfer the crisis to corresponding industries at the other side. The same stories happen if stock prices of a certain industry in one country drop, it will cause the drop of stock prices of the same industry in other countries. Furthermore the shock in real sector will intervene the capital market. If profit of an industry drops, people will not be interested to keep the stock of the industries. They will sell out and the prices of stock of the industry would fall.

Moreover ${ }^{10}$ concluded from his study that international trade is the most important determinant for the return of capital. So, crisis hire creates crisis there. The crisis in product market or crisis in international trade could create the crisis in financial market. The requirement of Market integration is free of significant restrictions; restrictions create sticky market which not freely reacts upon any change. This makes difficulty for the realization of market integration. It seems that the integration could only happen in case of common factor where all markets move due to the same cause at the same time. The common factor could move all markets at the same or reverse direction 11,12 .

The second theory is the theory of contagion effect, which ${ }^{3}$ define the contagion as the spread of shock from one economy to others. In ${ }^{13}$ state that contagion is caused by massive speculators' action in one country at a same short period. Define contagion as shock which related to crisis ${ }^{\text {A }}$. Analytically make a difference between shock which brings large change in a short time and spillover which affects slowly. Deeply in contagion theory, analytically divide the contagion theories into two basic

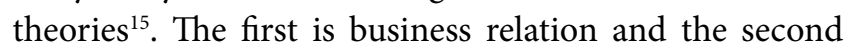

is investors' behavior. The business relation is related to complicated real sector economic relationship such trade, finance etc. It there is crisis in real sector, the crisis will move into financial sector and hence into capital markets. It follows the line of market integration theory. The later is not economic but psychological behavioral. It is related to subjective judgment onto the current phenomenon. It is heavily affected by asymmetric information, and the attitude toward risks. Since there is asymmetric information, investors tend to behave as leader and follower. Investors that have enough information will act as leader and tend to react earlier while others that have less information tend act as followers.

In relation with leader-follower up call" hypothesis. Since information is expensive, many investors just follow the decision made by well informed investors. Moreover if the crisis occurs in other country, investors that do not have enough information about financial situation in the country tend to assume that the crisis could happen soon in our own country. Due to the assumption, they make the decision. More often investors in small country just follow what investors in developed countries have done. Similar to 1 claim that there are 2 groups of investors; one is well informed and the other is less informed. Both groups tend to find out more information. If the well informed know that there would be crisis, they withdraw their money from one country, the less informed group tends to abandon their information and follow the behavior of the well informed. This causes the problem becomes more serious when the action quickly develops from individual to massive group's behaviour ${ }^{\text {1月. }}$.

The theory related to impact of crisis towards other economies, or shock transferring, has been known before the World War II. However since the crises existed in Mexico in late 1994 and early 1995 and in Thailand 1997 which was then expanded to become Asian Crisis, the term of contagion has been discussed frequently. In fif $^{2}$ study, classifies it into 3 types: the first is "monsoonal effects", attributed The second is "spillover Effect". And the third is "Contagious Effect". In of contagion operating through changes in expectations but not through the change in values of a country's fundamentals. Thus the crisis affects the situation in other markets or other countries by constituted a "wake up call". 


\section{Methodologies}

Capital markets are characterized by up and down change in stock prices. Every moment, prices of stock change. All market change whether they are related or not. This fact triggers an important question of how to identify if two markets are related or if they are not related. The degree of financial market integration would be easily investigated the relationship among the rates of return among the markets 1 . The integration in financial market could be seen at the discount rate differentials among the markets 1 . If the differentials tend to be increasingly smaller, it could be claimed that the integration is increasingly strong. Basically, the discount rate among markets can be use as indicators for risk and return 1 . Market Integration can also be seen statistically from correlation among them from time to time ${ }^{\text {月. }}$. The change in prices creates capital loss or gain, in other words, it gives return. The price movement in some certain range each day, traders still treats it as normal. However if it goes further (upper or lower) out of the range, they call abnormal. If there is a shock (abnormal returns) in a capital market, and other markets will show following abnormal returns, then it could possibly exists relation among the markets.

\subsection{Model}

A fully integration is well explained by Structural Vector Auto Regression (SVAR):

$A_{0} Y_{t}=A_{1} Y_{t-1}+\ldots+A_{k} Y_{t-k}+B_{1} X_{t-1}+\ldots+B_{h} X_{t-h}+\Phi E_{t}$

$Y_{t}=A_{0}^{-1} A_{1} Y_{t-1}+\ldots+A_{0}^{-1} A_{k} Y_{t-k}+A_{0}^{-1} B_{1} X_{t-1}+\ldots+A_{0}^{-1} B_{h} X_{t-h}+A_{0}^{-1} \Phi E_{t}$

$Y_{t}=A_{1}^{*} Y_{t-1}+\ldots+A_{k}^{*} Y_{t-k}+B_{1}^{*} X_{t-1}+\ldots+B_{h}^{*} X_{t-h}+Z$

$\theta \mathrm{Z}_{\mathrm{t}}=\Phi \mathrm{E}_{\mathrm{t}}$

$\theta=\mathrm{A}_{0} ; \Phi=\mathrm{I}$

$Y=[J K S E$ KLSE STI SET PSE $]$

$X=[\text { KS11 N255 HSI DJIA }]^{\prime}$

$\mathrm{Y}$ and $\mathrm{X}$ are vector of Stock indices in ASEAN region and of indices of Global Stock markets (South Korea, Japan, China, and US), respectively. It is based on Abnormal Returns in Range (ARR).The Abnormal Returns in Range can be defined as returns situated outside the normal range of daily stock prices movement. So, it can be written, the normal returns, as:

$R=\frac{p_{t}-p_{t-1}}{p_{t-1}}$
Abnormal Return in Point (ARP):

$A R P=R-\widehat{R}$

where $\hat{R}$ is the expected return

Abnormal Return in Range (ARR) (see in ${ }^{5}$ ):

$A R R\left\{\begin{array}{lll}=A R P & \text { if } & U \leq R \leq L \\ =0 & & \text { otherwise }\end{array}\right.$

Where $U$ is upper bound of the normal range and $\mathrm{L}$ is the Lower bound. Matrices $\mathrm{A}(\mathrm{s})$ and $\mathrm{B}(\mathrm{s})$ are of parameters of the ASEAN and of global Stock markets respectively. E is Matrix of the residual of Structural Form and $\mathrm{Z}$ is Matrix of residual of the Reduced Form. Parameter Matrix A0 will show the pattern of integration at current time, while Parameter Matrix A1 will show the pattern of integration at time $\mathrm{t}-1$ and so on. By analyzing corresponding elements of different matrixs $\mathrm{A}(\mathrm{s})$, the change in the pattern over time, as well as the consistency among different subsamples can be identified. It was hypothesized that it would be a consistent Pattern of integration. The Hypothesis $\left(\mathrm{H}_{1}\right)$ will be true if all corresponding parameters provided from different subsamples are still unchanged. Supposed that parameter $\mathrm{i}$ from subsample $\mathrm{i}$ is $\beta_{a i}$ and of subsample $\mathrm{b}$ is $\beta_{b i}$. The consistency exists if $\beta_{a i}=\beta_{b i}$ Or statistically it can be written as:

$H_{0}: \beta_{a i}-\beta_{b i}=0$ (Consistent)

$H_{1}: \beta_{a i}-\beta_{b i} \neq 0$ (Inconsistent)

The $t$ statistics for the difference between two-mean is used to test the hypothesis:

$t_{\beta a i-\beta b i}=\frac{\beta_{a i}-\beta_{b i}}{\sqrt{\sigma_{a i}^{2}+\sigma_{b i}^{2}}}$

In order to capture the structural Pattern at time 0 (current period), the model used is Structural Vector Auto Regression (SVAR). The crucial step in SVAR is to identify restriction. In case, such as in this study, where there is theoretical clue about structural relationship among variables, it needs to identify empirically. In this case researcher applies causality test (Engle-Granger causality) to make certain the direction of effect between two variables. To test consistencies, the model will be applied to two different sub-samples and the pattern identified from each sub sample will be compared. While the dynamics refers to changes of the pattern over time. 


\section{Results}

\subsection{Empirical Structural Pattern}

Sampling can be seen in Table 1-5. Since all stock markets are the same status, where they are interdependent theoretically, researcher has no clue to decide which one will act as cause and others the effect. It needs to test endogeneity to decide which one is exogenous or endogenous variable. This means that empirical test is appropriate. To test the structure, the study uses Englegranger causality test as given in Table 6 .

Table 1. Total sample received

\begin{tabular}{|c|c|c|c|c|}
\hline \multicolumn{3}{|c|}{ Structural VAR Estimates } & & \\
\hline \multirow{2}{*}{\multicolumn{4}{|c|}{\begin{tabular}{l|l} 
Date: $05 / 05 / 15$ Time: $21: 19$ & \\
Sample (adiusted): $1 / 11 / 200712 / 31 / 2014$
\end{tabular}}} & \\
\hline & & & & \\
\hline \multirow{2}{*}{\multicolumn{5}{|c|}{\begin{tabular}{|l|l|} 
Included observations: 2080 after adjustments & \\
Estimation method: method of scoring (analytic derivatives)
\end{tabular}}} \\
\hline & & & & \\
\hline \multicolumn{5}{|c|}{$\begin{array}{l}\text { Estimation method: method of scoring (analytic derivatives) } \\
\text { Convergence achieved after } 1 \text { iterations }\end{array}$} \\
\hline \multicolumn{4}{|c|}{\begin{tabular}{l|l} 
Structural VAR is just-identified \\
\end{tabular}} & \\
\hline \multicolumn{4}{|c|}{\begin{tabular}{l|l} 
Model: $\mathrm{Ae}=\mathrm{Bu}$ where $\mathrm{E}\left[\mathrm{uu}^{\prime}\right]=\mathrm{I}$ & \\
\end{tabular}} & \\
\hline \multicolumn{5}{|c|}{ Restriction Type: short-run pattern matrix } \\
\hline \multicolumn{2}{|c|}{$\mathrm{A}=$} & & & \\
\hline 1 & $C(2)$ & 0 & $C(5)$ & $\mathrm{C}(7)$ \\
\hline 0 & 1 & 0 & 0 & $\mathrm{C}(8)$ \\
\hline $\mathrm{C}(1)$ & $\mathrm{C}(3)$ & 1 & $\mathrm{C}(6)$ & $\mathrm{C}(9)$ \\
\hline 0 & $\mathrm{C}(4)$ & 0 & 1 & $\mathrm{C}(10)$ \\
\hline 0 & 0 & 0 & 0 & 1 \\
\hline \multicolumn{5}{|l|}{$B=$} \\
\hline $\mathrm{C}(11)$ & 0 & 0 & 0 & 0 \\
\hline 0 & $\mathrm{C}(12)$ & 0 & 0 & 0 \\
\hline 0 & 0 & $\mathrm{C}(13)$ & 0 & 0 \\
\hline 0 & 0 & 0 & $\mathrm{C}(14)$ & 0 \\
\hline \multirow[t]{2}{*}{0} & 0 & 0 & 0 & $C(15)$ \\
\hline & Coefficient & Std. Error & z-Statistic & Prob. \\
\hline $\mathrm{C}(1)$ & -0.225196 & 0.019557 & -11.51501 & 0.0000 \\
\hline $\mathrm{C}(2)$ & -0.356476 & 0.025901 & -13.76318 & 0.0000 \\
\hline $\mathrm{C}(3)$ & -0.268792 & 0.024130 & -11.13914 & 0.0000 \\
\hline $\mathrm{C}(4)$ & -0.351598 & 0.026759 & -13.13947 & 0.0000 \\
\hline $\mathrm{C}(5)$ & -0.269517 & 0.020394 & -13.21569 & 0.0000 \\
\hline $\mathrm{C}(6)$ & -0.256169 & 0.018938 & -13.52682 & 0.0000 \\
\hline $\mathrm{C}(7)$ & -0.148785 & 0.020298 & -7.330179 & 0.0000 \\
\hline $\mathrm{C}(8)$ & -0.247686 & 0.016775 & -14.76542 & 0.0000 \\
\hline$C(9)$ & -0.082181 & 0.018336 & -4.481921 & 0.0000 \\
\hline $\mathrm{C}(10)$ & -0.165855 & 0.021518 & -7.707759 & 0.0000 \\
\hline $\mathrm{C}(11)$ & 0.657992 & 0.010202 & 64.49806 & 0.0000 \\
\hline $\mathrm{C}(12)$ & 0.579685 & 0.008988 & 64.49806 & 0.0000 \\
\hline $\mathrm{C}(13)$ & 0.586878 & 0.009099 & 64.49806 & 0.0000 \\
\hline $\mathrm{C}(14)$ & 0.707446 & 0.010968 & 64.49806 & 0.0000 \\
\hline $\mathrm{C}(15)$ & 0.757714 & 0.011748 & 64.49806 & 0.0000 \\
\hline $\begin{array}{l}\text { Log like- } \\
\text { lihood }\end{array}$ & -10346.71 & & & \\
\hline
\end{tabular}

Restriction Matrix:

$\theta Z_{t}=\Phi E_{t}$

$\theta=\left[\begin{array}{ccccc}1 & N A & 0 & N A & N A \\ 0 & 1 & 0 & 0 & N A \\ N A & N A & 1 & N A & N A \\ 0 & N A & 0 & 1 & N A \\ 0 & 0 & 0 & 0 & 1\end{array}\right]$

$\Phi=I_{5.5}$

Figure 1 shows the trend of each capital market index in ASEAN region. The symbol of X01_JKSE, X02_KLSE, X03_STI, X04_SET and X05_PSE represent indexes of capital markets in Indonesia, Malaysia, Singapore, Thailand, and Philippines, respectively. They show a common pattern for some significant abnormal events such as crisis occurred in 2008, 2011 and 2013.

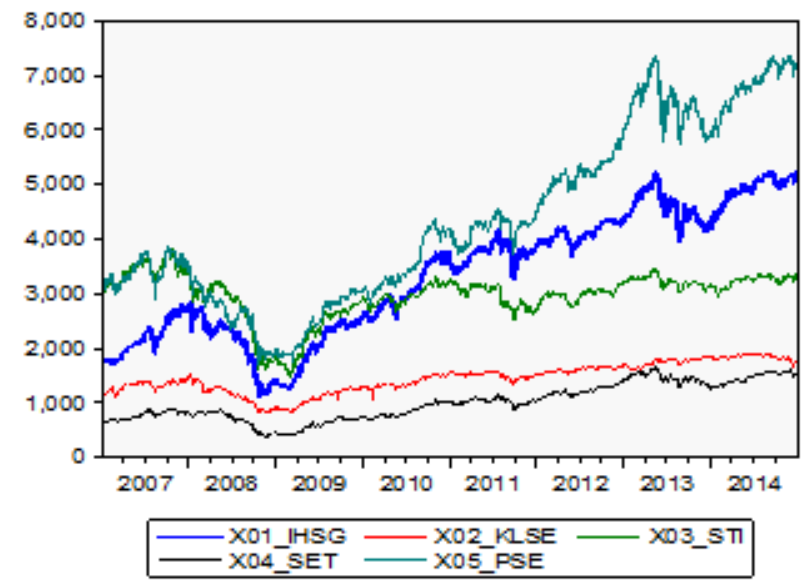

Figure 1. Indexes of ASEAN's capital markets.

Figure 2 shows the volatility of each market is shown. Capital market of Malaysia was relative less volatile than other 4 ASEAN capital markets. It is seen in the graph that capital market of Thailand is most volatile. Almost the same stories can be seen in Figure 3, where the abnormal return of each market was plotted. 
Table 2. Total sample

\begin{tabular}{|c|c|c|c|c|c|}
\hline \multicolumn{3}{|l|}{ Vector Autoregression Estimates } & & & \\
\hline \multicolumn{3}{|c|}{ Date: $07 / 22 / 16$ Time: $11: 41$} & & & \\
\hline \multicolumn{4}{|c|}{ Sample (adjusted): 1/11/2007 12/31/2014 } & & \\
\hline \multicolumn{4}{|c|}{ Included observations: 2080 after adjustments } & & \\
\hline \multicolumn{4}{|c|}{ Standard errors in ( ) \& t-statistics in [ ] } & & \\
\hline & X01 & $\mathrm{X} 02$ & X03 & X04 & X05 \\
\hline \multirow[t]{3}{*}{$\mathrm{X} 01(-1)$} & 0.066674 & 0.082515 & 0.006716 & 0.045687 & 0.063998 \\
\hline & $(0.02643)$ & $(0.02095)$ & $(0.02511)$ & $(0.02615)$ & $(0.02605)$ \\
\hline & {$[2.52243]$} & [3.93945] & {$[0.26745]$} & {$[1.74697]$} & [2.45699] \\
\hline \multirow[t]{3}{*}{$\mathrm{X} 01(-2)$} & -0.058344 & 0.004661 & -0.007305 & -0.010043 & 0.026367 \\
\hline & $(0.02654)$ & $(0.02103)$ & $(0.02521)$ & $(0.02626)$ & $(0.02615)$ \\
\hline & {$[-2.19846]$} & {$[0.22162]$} & {$[-0.28972]$} & {$[-0.38248]$} & {$[1.00821]$} \\
\hline \multirow[t]{3}{*}{$\mathrm{X} 02(-1)$} & -0.059937 & 0.038336 & -0.028013 & -0.013950 & 0.035462 \\
\hline & $(0.03257)$ & $(0.02581)$ & $(0.03094)$ & $(0.03222)$ & $(0.03209)$ \\
\hline & {$[-1.84031]$} & [1.48540] & {$[-0.90533]$} & {$[-0.43292]$} & [ 1.10491$]$ \\
\hline \multirow[t]{3}{*}{$\mathrm{X} 02(-2)$} & 0.000485 & -0.012098 & 0.018037 & -0.014373 & -0.005173 \\
\hline & $(0.03241)$ & $(0.02568)$ & $(0.03079)$ & $(0.03207)$ & $(0.03194)$ \\
\hline & {$[0.01496]$} & {$[-0.47106]$} & {$[0.58579]$} & {$[-0.44825]$} & {$[-0.16197]$} \\
\hline \multirow[t]{3}{*}{$\mathrm{X} 03(-1)$} & 0.017628 & 0.024735 & -0.003395 & -0.050151 & 0.126570 \\
\hline & $(0.02878)$ & $(0.02280)$ & $(0.02734)$ & $(0.02847)$ & $(0.02836)$ \\
\hline & {$[0.61259]$} & [ 1.08468$]$ & {$[-0.12419]$} & {$[-1.76144]$} & {$[4.46334]$} \\
\hline \multirow[t]{3}{*}{$\mathrm{X} 03(-2)$} & 0.043826 & 0.033760 & 0.008620 & 0.059170 & -0.001644 \\
\hline & $(0.02887)$ & $(0.02288)$ & $(0.02743)$ & $(0.02857)$ & $(0.02845)$ \\
\hline & {$[1.51791]$} & {$[1.47554]$} & {$[0.31423]$} & [2.07129] & {$[-0.05780]$} \\
\hline \multirow[t]{3}{*}{$\mathrm{X} 04(-1)$} & 0.033278 & 0.010427 & 0.022372 & 0.031623 & 0.069663 \\
\hline & $(0.02591)$ & $(0.02053)$ & $(0.02462)$ & $(0.02564)$ & $(0.02553)$ \\
\hline & {$[1.28424]$} & {$[0.50781]$} & {$[0.90877]$} & [ 1.23345$]$ & {$[2.72813]$} \\
\hline \multirow[t]{3}{*}{$\mathrm{X} 04(-2)$} & -0.010456 & -0.000228 & 0.013302 & -0.028680 & -0.039724 \\
\hline & $(0.02586)$ & $(0.02049)$ & $(0.02457)$ & $(0.02559)$ & $(0.02549)$ \\
\hline & {$[-0.40429]$} & {$[-0.01111]$} & {$[0.54136]$} & {$[-1.12084]$} & {$[-1.55864]$} \\
\hline \multirow[t]{3}{*}{$\mathrm{X} 05(-1)$} & -0.000267 & -0.001188 & 0.004334 & 0.059532 & 0.006918 \\
\hline & $(0.02415)$ & $(0.01914)$ & $(0.02295)$ & $(0.02390)$ & $(0.02380)$ \\
\hline & {$[-0.01106]$} & {$[-0.06206]$} & {$[0.18886]$} & [2.49113] & [0.29064] \\
\hline \multirow[t]{3}{*}{ X05(-2) } & 0.017269 & 0.028930 & 0.009874 & 0.031401 & -0.016185 \\
\hline & $(0.02371)$ & $(0.01879)$ & $(0.02253)$ & $(0.02346)$ & $(0.02337)$ \\
\hline & [0.72829] & [ 1.53964$]$ & {$[0.43830]$} & [ 1.33847] & {$[-0.69266]$} \\
\hline \multirow[t]{3}{*}{$\mathrm{C}$} & 0.054260 & -0.002105 & -0.003494 & 0.022416 & 0.037882 \\
\hline & $(0.01702)$ & $(0.01348)$ & $(0.01617)$ & $(0.01684)$ & $(0.01677)$ \\
\hline & [3.18874] & {$[-0.15611]$} & {$[-0.21614]$} & [ 1.33143$]$ & [2.25911] \\
\hline R-squared & 0.009942 & 0.026402 & 0.001717 & 0.011463 & 0.049780 \\
\hline Adj. R-squared & 0.005157 & 0.021696 & -0.003108 & 0.006685 & 0.045187 \\
\hline Sum sq. resids & 1223.247 & 768.1310 & 1104.093 & 1197.441 & 1187.875 \\
\hline S.E. equation & 0.768912 & 0.609309 & 0.730504 & 0.760758 & 0.757714 \\
\hline F-statistic & 2.077735 & 5.610681 & 0.355839 & 2.399236 & 10.83907 \\
\hline Log likelihood & -2399.298 & -1915.383 & -2292.715 & -2377.124 & -2368.782 \\
\hline Akaike AIC & 2.317595 & 1.852291 & 2.215111 & 2.296273 & 2.288252 \\
\hline Schwarz SC & 2.347422 & 1.882119 & 2.244938 & 2.326100 & 2.318080 \\
\hline Mean dependent & 0.055769 & 0.004327 & -0.001923 & 0.028365 & 0.043269 \\
\hline S.D. dependent & 0.770903 & 0.616028 & 0.729372 & 0.763314 & 0.775436 \\
\hline \multicolumn{2}{|c|}{ Determinant resid covariance (dof adj.) } & 0.014399 & & & \\
\hline \multicolumn{2}{|c|}{ Determinant resid covariance } & 0.014022 & & & \\
\hline \multicolumn{2}{|l|}{ Log likelihood } & -10319.13 & & & \\
\hline Akaike information criterion & & 9.975128 & & & \\
\hline Schwarz criterion & & 10.12427 & & & \\
\hline
\end{tabular}


Table 3. Sum sample 1

\begin{tabular}{|c|c|c|c|c|}
\hline \multicolumn{3}{|c|}{ Structural VAR Estimates } & & \\
\hline \multicolumn{5}{|c|}{ Date: $07 / 22 / 16$ Time: $11: 36$} \\
\hline \multicolumn{4}{|c|}{ Sample (adjusted): 1/11/2007 12/31/2009 } & \\
\hline \multicolumn{4}{|c|}{ Included observations: 776 after adjustments } & \\
\hline \multicolumn{5}{|c|}{ Estimation method: method of scoring (analytic derivatives) } \\
\hline \multicolumn{4}{|c|}{ Convergence achieved after 1 iterations } & \\
\hline \multicolumn{3}{|c|}{ Structural VAR is just-identified } & & \\
\hline \multicolumn{3}{|c|}{ Model: $\mathrm{Ae}=\mathrm{Bu}$ where $\mathrm{E}[\mathrm{uu}]=\mathrm{I}$} & & \\
\hline \multicolumn{4}{|c|}{ Restriction Type: short-run pattern matrix } & \\
\hline \multicolumn{2}{|c|}{$\mathrm{A}=$} & & & \\
\hline 1 & $\mathrm{C}(2)$ & 0 & $\mathrm{C}(5)$ & $\mathrm{C}(7)$ \\
\hline 0 & 1 & 0 & 0 & $\mathrm{C}(8)$ \\
\hline $\mathrm{C}(1)$ & $\mathrm{C}(3)$ & 1 & $\mathrm{C}(6)$ & $\mathrm{C}(9)$ \\
\hline 0 & $\mathrm{C}(4)$ & 0 & 1 & $\mathrm{C}(10)$ \\
\hline 0 & 0 & 0 & 0 & 1 \\
\hline \multicolumn{2}{|l|}{$\mathrm{B}=$} & & & \\
\hline $\mathrm{C}(11)$ & 0 & 0 & 0 & 0 \\
\hline 0 & $\mathrm{C}(12)$ & 0 & 0 & 0 \\
\hline 0 & 0 & $\mathrm{C}(13)$ & 0 & 0 \\
\hline 0 & 0 & 0 & $\mathrm{C}(14)$ & 0 \\
\hline \multirow[t]{2}{*}{0} & 0 & 0 & 0 & $\mathrm{C}(15)$ \\
\hline & Coefficient & Std. Error & z-Statistic & Prob. \\
\hline $\mathrm{C}(1)$ & -0.262515 & 0.032845 & -7.992642 & 0.0000 \\
\hline $\mathrm{C}(2)$ & -0.328415 & 0.040064 & -8.197151 & 0.0000 \\
\hline $\mathrm{C}(3)$ & -0.322432 & 0.038211 & -8.438222 & 0.0000 \\
\hline $\mathrm{C}(4)$ & -0.406293 & 0.039414 & -10.30826 & 0.0000 \\
\hline$C(5)$ & -0.307031 & 0.034222 & -8.971728 & 0.0000 \\
\hline$C(6)$ & -0.278194 & 0.032895 & -8.456962 & 0.0000 \\
\hline $\mathrm{C}(7)$ & -0.093683 & 0.033407 & -2.804268 & 0.0050 \\
\hline $\mathrm{C}(8)$ & -0.283726 & 0.029981 & -9.463520 & 0.0000 \\
\hline $\mathrm{C}(9)$ & -0.056305 & 0.030720 & -1.832846 & 0.0668 \\
\hline $\mathrm{C}(10)$ & -0.122625 & 0.034765 & -3.527201 & 0.0004 \\
\hline $\mathrm{C}(11)$ & 0.698982 & 0.017743 & 39.39543 & 0.0000 \\
\hline $\mathrm{C}(12)$ & 0.667795 & 0.016951 & 39.39543 & 0.0000 \\
\hline $\mathrm{C}(13)$ & 0.639530 & 0.016234 & 39.39543 & 0.0000 \\
\hline $\mathrm{C}(14)$ & 0.733210 & 0.018612 & 39.39543 & 0.0000 \\
\hline$C(15)$ & 0.799588 & 0.020296 & 39.39543 & 0.0000 \\
\hline Log likelihood & -4152.985 & & & \\
\hline
\end{tabular}


Table 4. Received vs. Subsample 1

\begin{tabular}{|c|c|c|c|c|c|}
\hline \multicolumn{3}{|l|}{ Vector Autoregression Estimates } & & & \\
\hline \multicolumn{3}{|c|}{ Date: $07 / 22 / 16$ Time: $11: 37$} & & & \\
\hline \multicolumn{6}{|c|}{ Sample (adjusted): 1/11/2007 12/31/2009 } \\
\hline \multicolumn{6}{|c|}{ Included observations: 776 after adjustments } \\
\hline \multicolumn{6}{|c|}{ Standard errors in ( ) \& t-statistics in [ ] } \\
\hline & $\mathrm{X} 01$ & $\mathrm{X} 02$ & $\mathrm{X} 03$ & $\mathrm{X} 04$ & $\mathrm{X} 05$ \\
\hline \multirow[t]{3}{*}{$\mathrm{X} 01(-1)$} & 0.116944 & 0.159051 & 0.041947 & 0.080270 & 0.148612 \\
\hline & $(0.04406)$ & $(0.03785)$ & $(0.04489)$ & $(0.04318)$ & $(0.04291)$ \\
\hline & {$[2.65440]$} & {$[4.20243]$} & {$[0.93436]$} & {$[1.85905]$} & [3.46347] \\
\hline \multirow[t]{3}{*}{$\mathrm{X} 01(-2)$} & -0.040985 & -0.002550 & -0.041464 & 0.039213 & 0.032680 \\
\hline & $(0.04462)$ & $(0.03833)$ & $(0.04547)$ & $(0.04373)$ & $(0.04345)$ \\
\hline & {$[-0.91858]$} & {$[-0.06654]$} & {$[-0.91199]$} & [0.89677] & [0.75205] \\
\hline \multirow[t]{3}{*}{$\mathrm{X} 02(-1)$} & -0.040388 & 0.058096 & 0.053995 & 0.009602 & 0.069232 \\
\hline & $(0.05107)$ & $(0.04387)$ & $(0.05204)$ & $(0.05005)$ & $(0.04974)$ \\
\hline & {$[-0.79083]$} & [ 1.32421$]$ & [ 1.03757$]$ & {$[0.19185]$} & [ 1.39193$]$ \\
\hline \multirow[t]{3}{*}{$\mathrm{X} 02(-2)$} & -0.010013 & -0.034117 & -0.015663 & -0.076929 & -0.054745 \\
\hline & $(0.05062)$ & $(0.04349)$ & $(0.05158)$ & $(0.04961)$ & $(0.04930)$ \\
\hline & {$[-0.19782]$} & {$[-0.78457]$} & {$[-0.30365]$} & {$[-1.55067]$} & {$[-1.11045]$} \\
\hline \multirow[t]{3}{*}{$\mathrm{X} 03(-1)$} & 0.037009 & 0.008008 & -0.029624 & -0.081165 & 0.069487 \\
\hline & $(0.04628)$ & $(0.03976)$ & $(0.04716)$ & $(0.04536)$ & $(0.04508)$ \\
\hline & {$[0.79963]$} & {$[0.20142]$} & {$[-0.62813]$} & {$[-1.78936]$} & [ 1.54153$]$ \\
\hline \multirow[t]{3}{*}{$\mathrm{X} 03(-2)$} & 0.015421 & 0.019208 & -0.024043 & 0.033737 & -0.018636 \\
\hline & $(0.04627)$ & $(0.03975)$ & $(0.04715)$ & $(0.04534)$ & $(0.04506)$ \\
\hline & {$[0.33330]$} & {$[0.48328]$} & {$[-0.50997]$} & {$[0.74404]$} & {$[-0.41359]$} \\
\hline \multirow[t]{3}{*}{$\mathrm{X} 04(-1)$} & 0.020506 & -0.021407 & -0.033124 & 0.030356 & 0.051634 \\
\hline & $(0.04432)$ & $(0.03807)$ & $(0.04516)$ & $(0.04344)$ & $(0.04316)$ \\
\hline & {$[0.46270]$} & {$[-0.56227]$} & {$[-0.73347]$} & {$[0.69887]$} & [ 1.19623$]$ \\
\hline \multirow[t]{3}{*}{\begin{tabular}{|l}
$\mathrm{X} 04(-2)$ \\
\end{tabular}} & -0.029314 & 0.011544 & 0.038190 & -0.015519 & -0.063089 \\
\hline & $(0.04418)$ & $(0.03795)$ & $(0.04502)$ & $(0.04330)$ & $(0.04303)$ \\
\hline & {$[-0.66349]$} & {$[0.30416]$} & [0.84827] & {$[-0.35842]$} & {$[-1.46618]$} \\
\hline \multirow[t]{3}{*}{$\mathrm{X} 05(-1)$} & -0.024536 & -0.008827 & -0.002733 & 0.040609 & -0.032015 \\
\hline & $(0.03952)$ & $(0.03395)$ & $(0.04027)$ & $(0.03873)$ & $(0.03849)$ \\
\hline & {$[-0.62081]$} & {$[-0.25997]$} & {$[-0.06786]$} & [1.04840] & {$[-0.83172]$} \\
\hline \multirow[t]{3}{*}{$\mathrm{X} 05(-2)$} & 0.044871 & 0.114327 & 0.050353 & 0.044335 & -0.006392 \\
\hline & $(0.03880)$ & $(0.03333)$ & $(0.03954)$ & $(0.03803)$ & $(0.03779)$ \\
\hline & [ 1.15646$]$ & [ 3.42996] & [ 1.27355$]$ & {$[1.16590]$} & {$[-0.16916]$} \\
\hline \multirow[t]{3}{*}{$\mathrm{C}$} & 0.053690 & 0.001747 & -0.000702 & 0.001758 & 0.017712 \\
\hline & $(0.02967)$ & $(0.02549)$ & $(0.03023)$ & $(0.02908)$ & $(0.02890)$ \\
\hline & [ 1.80963$]$ & {$[0.06855]$} & {$[-0.02322]$} & {$[0.06045]$} & [0.61296] \\
\hline R-squared & 0.020452 & 0.060073 & 0.006679 & 0.017184 & 0.070922 \\
\hline Adj. R-squared & 0.007647 & 0.047786 & -0.006305 & 0.004337 & 0.058778 \\
\hline Sum sq. resids & 515.6248 & 380.5240 & 535.3986 & 495.2581 & 489.0959 \\
\hline S.E. equation & 0.820987 & 0.705278 & 0.836581 & 0.804609 & 0.799588 \\
\hline F-statistic & 1.597226 & 4.889273 & 0.514398 & 1.337581 & 5.839726 \\
\hline Log likelihood & -942.4924 & -824.6062 & -957.0936 & -926.8558 & -921.9979 \\
\hline Akaike AIC & 2.457455 & 2.153624 & 2.495087 & 2.417154 & 2.404634 \\
\hline Schwarz SC & 2.523428 & 2.219598 & 2.561060 & 2.483128 & 2.470608 \\
\hline Mean dependent & 0.057990 & 0.014175 & 0.001289 & 0.010309 & 0.027062 \\
\hline S.D. dependent & 0.824144 & 0.722758 & 0.833956 & 0.806360 & 0.824176 \\
\hline \multicolumn{2}{|c|}{ Determinant resid covariance (dof adj.) } & 0.030629 & & & \\
\hline \multicolumn{2}{|c|}{ Determinant resid covariance } & 0.028519 & & & \\
\hline \multicolumn{2}{|l|}{ Log likelihood } & -4125.288 & & & \\
\hline Akaike information criterion & & 10.77394 & & & \\
\hline Schwarz criterion & & 11.10381 & & & \\
\hline
\end{tabular}


Table 5. Sub sample 2

\begin{tabular}{|c|c|c|c|c|}
\hline \multicolumn{3}{|c|}{ Structural VAR Estimates } & & \\
\hline \multicolumn{3}{|c|}{ Date: $07 / 22 / 16$ Time: $11: 39$} & & \\
\hline \multicolumn{3}{|c|}{ Sample: $1 / 11 / 201012 / 31 / 2014$} & & \\
\hline \multicolumn{3}{|c|}{ Included observations: 1298} & & \\
\hline \multicolumn{5}{|c|}{ Estimation method: method of scoring (analytic derivatives) } \\
\hline \multicolumn{4}{|c|}{ Convergence achieved after 1 iterations } & \\
\hline \multicolumn{3}{|c|}{ Structural VAR is just-identified } & & \\
\hline \multicolumn{3}{|c|}{ Model: $\mathrm{Ae}=\mathrm{Bu}$ where $\mathrm{E}\left[\mathrm{uu} \mathbf{u}^{\prime}\right]=\mathrm{I}$} & & \\
\hline \multicolumn{4}{|c|}{ Restriction Type: short-run pattern matrix } & \\
\hline \multicolumn{2}{|c|}{$\mathrm{A}=$} & & & \\
\hline 1 & $\mathrm{C}(2)$ & 0 & $\mathrm{C}(5)$ & $\mathrm{C}(7)$ \\
\hline 0 & 1 & 0 & 0 & $\mathrm{C}(8)$ \\
\hline $\mathrm{C}(1)$ & $\mathrm{C}(3)$ & 1 & $\mathrm{C}(6)$ & $\mathrm{C}(9)$ \\
\hline 0 & $\mathrm{C}(4)$ & 0 & 1 & $\mathrm{C}(10)$ \\
\hline 0 & 0 & 0 & 0 & 1 \\
\hline \multicolumn{5}{|l|}{$\mathrm{B}=$} \\
\hline $\mathrm{C}(11)$ & 0 & 0 & 0 & 0 \\
\hline 0 & $\mathrm{C}(12)$ & 0 & 0 & 0 \\
\hline 0 & 0 & $\mathrm{C}(13)$ & 0 & 0 \\
\hline 0 & 0 & 0 & $\mathrm{C}(14)$ & 0 \\
\hline \multirow[t]{2}{*}{0} & 0 & 0 & 0 & $\mathrm{C}(15)$ \\
\hline & Coefficient & Std. Error & z-Statistic & Prob. \\
\hline $\mathrm{C}(1)$ & -0.198836 & 0.024099 & -8.250751 & 0.0000 \\
\hline $\mathrm{C}(2)$ & -0.382042 & 0.034695 & -11.01139 & 0.0000 \\
\hline $\mathrm{C}(3)$ & -0.204388 & 0.031499 & -6.488656 & 0.0000 \\
\hline $\mathrm{C}(4)$ & -0.304960 & 0.037139 & -8.211402 & 0.0000 \\
\hline $\mathrm{C}(5)$ & -0.242189 & 0.025282 & -9.579529 & 0.0000 \\
\hline $\mathrm{C}(6)$ & -0.237499 & 0.022713 & -10.45632 & 0.0000 \\
\hline $\mathrm{C}(7)$ & -0.189739 & 0.025562 & -7.422608 & 0.0000 \\
\hline $\mathrm{C}(8)$ & -0.220553 & 0.019659 & -11.21911 & 0.0000 \\
\hline $\mathrm{C}(9)$ & -0.108610 & 0.022660 & -4.792959 & 0.0000 \\
\hline $\mathrm{C}(10)$ & -0.192732 & 0.027550 & -6.995837 & 0.0000 \\
\hline $\mathrm{C}(11)$ & 0.630375 & 0.012372 & 50.95096 & 0.0000 \\
\hline $\mathrm{C}(12)$ & 0.517237 & 0.010152 & 50.95096 & 0.0000 \\
\hline $\mathrm{C}(13)$ & 0.547316 & 0.010742 & 50.95096 & 0.0000 \\
\hline $\mathrm{C}(14)$ & 0.692073 & 0.013583 & 50.95096 & 0.0000 \\
\hline $\mathrm{C}(15)$ & 0.730293 & 0.014333 & 50.95096 & 0.0000 \\
\hline Log likelihood & -6086.184 & & & \\
\hline
\end{tabular}

Table 6. Pattern of causality among ASEAN indices

\begin{tabular}{|c|c|c|}
\hline Indices & Causal Effect & Indices \\
\hline Indonesia (JKSE) & & Malaysia (KLSE) \\
\hline Indonesia (JKSE) & & Singapore (STI) \\
\hline Indonesia (JKSE) & & Thailand (SET) \\
\hline Indonesia (JKSE) & & Philippines (PSI) \\
\hline Malaysia (KLSE) & & Singapore (STI) \\
\hline Malaysia (KLSE) & & Thailand (SET) \\
\hline Malaysia (KLSE) & & Philippines (PSI) \\
\hline Singapore (STI) & & Thailand (SET) \\
\hline Singapore (STI) & & Philippines (PSI) \\
\hline Thailand (SET) & & Philippines (PSI) \\
\hline
\end{tabular}

Source: Result from Engle-Granger Causalities test 
Table 7. Consistency result

\begin{tabular}{|l|c|c|}
\hline \multicolumn{3}{|c|}{ Hypothesis testing at Current time Using } \\
Equation $t_{\beta a i-\beta b i}=\frac{\beta_{a i}-\beta_{b i}}{\sqrt{\sigma_{a i}^{2}+\sigma_{b i}^{2}}}$ \\
\hline Parameters & $\mathbf{t}_{\text {est }}$ & Concl. \\
\hline$\beta_{\mathrm{a} 1} \leftrightarrow \beta_{\mathrm{b} 1}$ & 1.012 & Consist \\
\hline$\beta_{\mathrm{a} 2} \leftrightarrow \beta_{\mathrm{b} 2}$ & -1.563 & Consist \\
\hline$\beta_{\mathrm{a} 3} \leftrightarrow \beta_{\mathrm{b} 3}$ & -1.524 & Consist \\
\hline$\beta_{\mathrm{a} 4} \leftrightarrow \beta_{\mathrm{b} 4}$ & $2.284^{\star}$ & Consist \\
\hline$\beta_{\mathrm{a} 5} \leftrightarrow \beta_{\mathrm{b} 5}$ & $-2.384^{*}$ & Consist \\
\hline$\beta_{\mathrm{a} 6} \leftrightarrow \beta_{\mathrm{b} 6}$ & -1.871 & Consist \\
\hline$\beta_{\mathrm{a} 7} \leftrightarrow \beta_{\mathrm{b} 7}$ & -1.762 & Consist \\
\hline$\beta_{\mathrm{a} 8} \leftrightarrow \beta_{\mathrm{b} 8}$ & -1.018 & Consist \\
\hline$\beta_{\mathrm{a} 9} \leftrightarrow \beta_{\mathrm{b} 9}$ & 1.370 & Consist \\
\hline$\beta_{\mathrm{a} 10} \leftrightarrow \beta_{\mathrm{b} 10}$ & 1.580 & Consist \\
\hline
\end{tabular}

\subsection{Pattern Consistency}

By using statistical test for the difference between two means (see equation (6)) the consistency of the pattern can be investigated from Table 7 .

The pattern of integration among ASEAN 5 Capital markets is compared between first subsample $\left(11^{\text {th }}\right.$ Jan
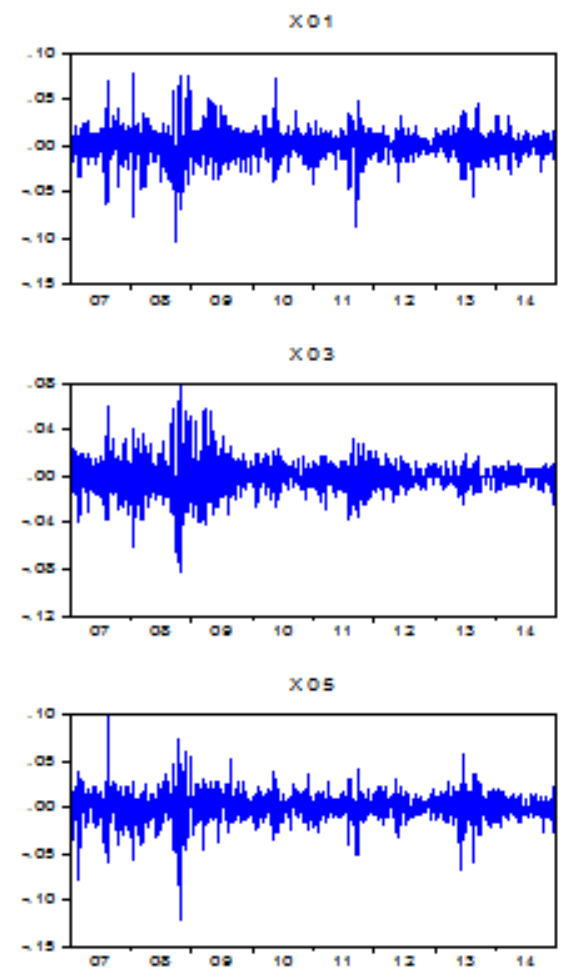

Figure 2. Volatility in ASEAN's capital markets.
2007 to $31^{\text {st }}$ Dec 2009) and the second subsample ( $11^{\text {th }}$ Jan 2010 to $31^{\text {st }}$ Dec 2014). Identification the structure of the relationship among the ASEAN markets based on Engel-Granger causalities of the two periods provide the same structure. Empirical is structure still unchanged even though the distance between the periods is quite far. This is an indication that the empirical structure is consistent. It also has the consistency in direction or arrow. Furthermore the consistency is tested by $t$ test of difference between two means. The results show that almost all parameters have no difference between both periods, except 2 parameters $\beta_{4}$ and $\beta_{5}$.

The $\beta_{4}$, represents the relationship between Indonesia Stock market and the Philippines'. Even though the sign is still the unchanged, the magnitude tend to be higher from -0.0937 to -0.1897 . While the $\beta_{5}$, represents the relationship between Singapore Stock market and the Malaysia's. Even though the sign is still the unchanged, the magnitude tends to be smaller from -0.40629 to -0.30496 . Thus the two pairs of markets could be said as weak consistent. However, the overall structure can be said as consistent.
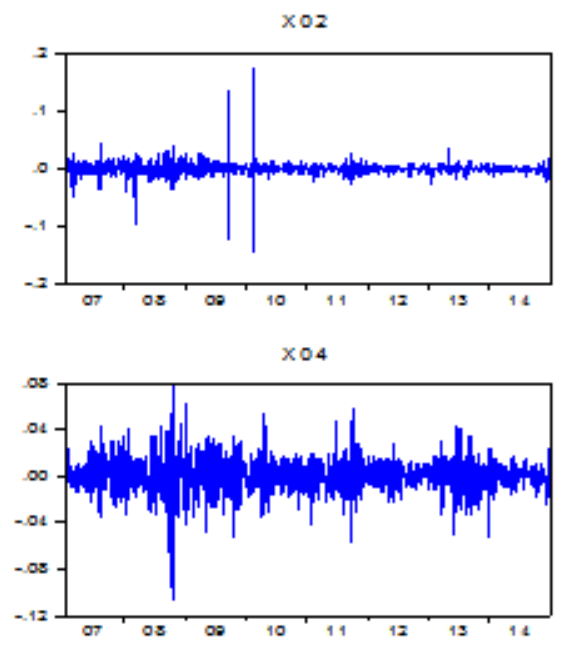

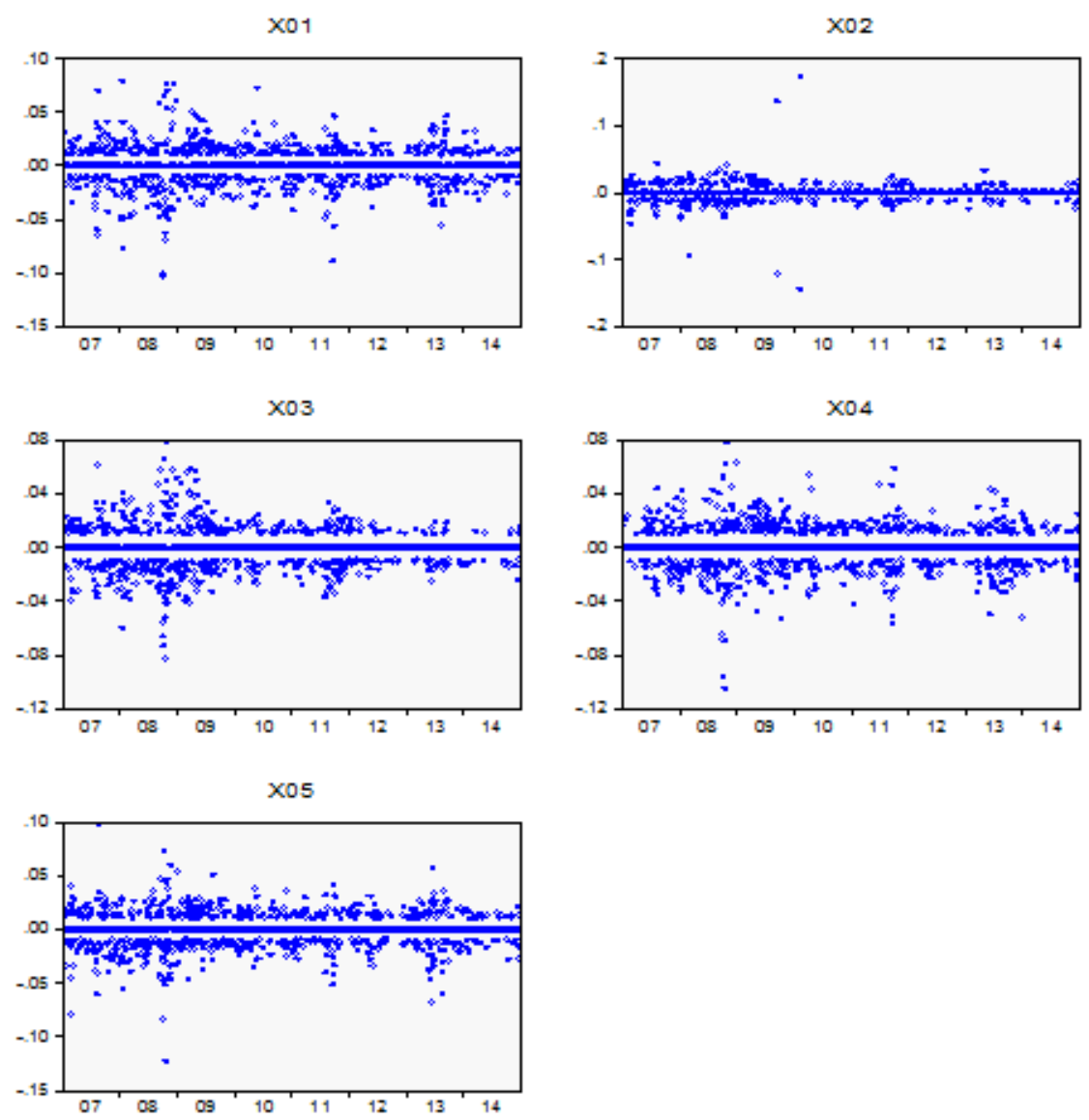

Figure 3. Abnormal return in ASEAN's capital markets.
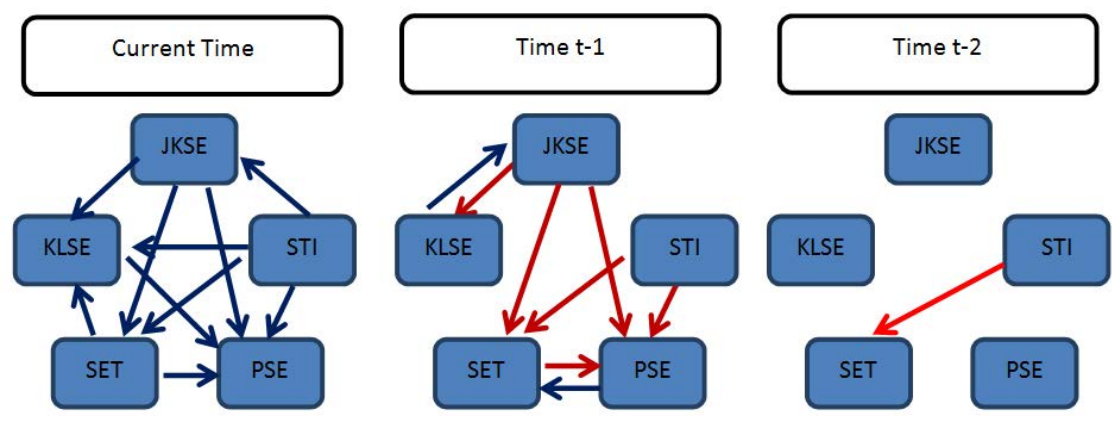

Figure 4. Dynamics of the pattern.

\subsection{The Structure Dynamics}

The dynamics means the changes over time. The dynamics of the structure means how the structure varies from period to period. Figure 4 shows how the pattern of the integration among ASEAN stock markets change from time 0 compare that of time t- 1 and the periods before. It is seen that relationship at current period is more complicated compare to the one period lag and the second period lag. At the current period, all markets are integrated either as transmitter and receiver the shock. The effect of the shock for the period after is not so complete. There is some relationship still hold but some others disappear. However there are 2 pairs of market that look no relation at the current period of the schlock, but the effect appears after one day; Shock in Malaysia has effect to Indonesian market after a day (one lag). The 
same story happens for Philippines to Thailand. Almost all effect disappears after one lag except the relation between Singaporean market and Thailand market. The effect in the relation lasts from current period, one day lag and two-day lag. What we can say from this dynamics is that the reaction in stock market is quick and lasts for 2 days. It changes drastically in days.

\section{Conclusion and Recommendations}

The integration is almost fully achieved. Except the relation between Philippines' capital Market and the Singapore's capital market, all other markets are fully integrated significantly. The integration exists at time zero (0), while at lag 1 and lag 2 periods it reduces sharply. The intervention of global capital markets has been controlled, thus the integration identified is purely the relation among the ASEAN's markets. This findings guide policy makers to pay more attention to any abnormal change in any ASEAN capital markets. A shock in any ASEAN market will easily and quickly affects all other markets in ASEAN region. Even though the size of the impact is variably, but the impact surely will exist significantly. The care should be more on the markets that have both direction and single direction impact.

\section{References}

1. Banerjee A. A simple model of herd behavior. Quarterly Journal of Economics. 1992; 107:797-817.

2. Bikhchandani S, Hirshleifer D, Welch I. A theory of fads, fashion, custom and cultural changes as information cascades. Journal of Political Economy. 1992; 100(5):992-1020.

3. Dornbusch R, Yung C, Claessens S. Contagion: Understanding how it spreads. The World Bank Research Observer. $2000 ; 15(2): 177-97$.

4. Forbes K. Measuring Contagion: Conceptual and empirical issues. The Contagion Conference on International Financial Contagion; 2000. p. 43-66.

5. Goldstein M, Kaminsky G, Reinhart C. Assessing Financial Vulnerability: An Early Warning System for Emerging Markets. Washington, D.C.: Institute for International Economics; 1998. p. 150.

6. Kaminsky G, Reinhart C. Financial crisis in Asia and Latin America: Then and now. American Economic Review. 1998; 88:444-8.

7. Kaminsky GL, Reinhart CM, Vegh CA. The unholy trinity of financial contagion. Journal of Economic Perspectives: American Economic Association. 2003; 17(4):51-74.

8. Kaneta K. The Integration of Capital Markets: A Study of Market Integration Principles and the Effects of Macroeconomic Shocks on National Stock Market Comovements [Honor Project]. Illinois: Wesleyan University; 2000. p. 1-29.

9. Karolyi GA, Stulz R. Why Do Markets Move Together? An investigation of U.S.-Japan stock return co movements. The Journal of Finance. 1996:1-50.

10. Kasa K. Comovements among national stock markets. Federal Reserve Bank of San Francisco Economic Review. 1995:14-20.

11. Kasa K. Borrowing constraints and asset market dynamics: Evidence from the Pacific Basin. Federal Reserve Bank of San Francisco Economic Review. 1998:17-28.

12. Korajczyk RA. A measure of stock market integration for developed and emerging markets. The World Bank Economic Review. 1996; 10(2):267-89.

13. Masson P. Contagion: Monsoonal effects, spillovers and jumps between multiple equilibrium. IMF Working Paper WP/98/142 International Monetary Fund; Washington, D.C. 1999. p. 1-32.

14. Murphy JJ. Inter-market Technical Analysis: Trading Strategies for the Global Stock, Bond, Commodity and Currency Markets. John Wiley \& Sons, Inc; 1991. p. 288.

15. Shiller R. Conversation, information and herd behavior. American Economic Review. 1995; 85(2):181-5.

16. Tamirisa N. Exchange and capital controls as barriers to trade. International Monetary Fund, Monetary and Exchange Affairs Department. 1998:1-19.

17. Wongbangpo P, Subhash SC. Stock market and macroeconomic fundamental dynamic integration: ASEAN countries. Southern Illinois University; 2000. p. 27-51. 